\title{
Ventricular tachycardia-like electrocardiographic artifact on total thyroidectomy
}

\author{
Yong Sung Cho, Ji Yeon Kim, Kyung Woo Kim, Jun Hyun Kim, and Won Joo Choe
}

Department of Anesthesiology and Pain Medicine, Inje University, Ilsan Paik Hospital, Goyang, Korea

Electrocardiography (ECG) is an essential monitoring method that provides important information related to cardiac physiology. However, the sinus rhythm can be interpreted as a serious arrhythmia, such as ventricular tachycardia (VT), due to artifacts [1]. We present a case in which a VT-like artifact appeared on an ECG during a total thyroidectomy.

A 33-year-old female was admitted for a thyroidectomy due to hyperthyroidism. There were no significant findings on a preoperational examination, including thyroid function test. The ECG showed a normal sinus rhythm. The thyroid was significantly enlarged overall; the sizes of the right lobe, isthmus, and left lobe were $7 \times 3 \times 3 \mathrm{~cm}, 5 \times 1.5 \times 1 \mathrm{~cm}$, and $6.5 \times 3.5 \times 2 \mathrm{~cm}$, respectively.

Initial vital signs were blood pressure (BP) of 120/70 $\mathrm{mmHg}$, pulse rate (PR) of $70 / \mathrm{min}$, body temperature of $36.5^{\circ} \mathrm{C}$, and $\mathrm{SaO}_{2}$ of $98 \%$. ECG leads were attached to both infraspinatus muscle area and lateral side of left $4^{\text {th }}$ rib. Anesthesia was induced with propofol, rocuronium and remifentanil. After the endotracheal intubation, anesthesia maintained with sevoflurane, remifentanil. An electrocautery grounding pad was attached to the left peroneal posterior surface, and electrical noise was not observed.

The surgery proceeded, but 170 minutes into the operation, when the operator retracted the upper pole for dissection, the PR increased to above $190 / \mathrm{min}$ with a VT-like ECG rhythm. At that time, BP was $110 / 70 \mathrm{mmHg}$. We asked for an immediate suspension of surgery and identified it as an arrhythmia related to thyroid disease. Esmolol $30 \mathrm{mg}$ was administered, and then amiodarone and direct current cardioversion were prepared to address the VT. Immediately after the administration, the PR fell to within the normal range of 70-80 / $\mathrm{min}$ and normal QRS rhythm was restored. However, a similar manifestation on the ECG and tachycardia were seen 15 minutes later on the same surgical procedure. At that time, the patient presented a BP of 103/63 mmHg, PR of $74 / \mathrm{min}$, and normal pulse oximeter wave was observed. On analyzing the ECG rhythm strip printed from the monitor, the hidden QRS wave was found within the waveform suspected as artifacts (Fig. 1). The surgery was continued without further treatment, and completed without any problem. The patient presented a stable hemodynamic status and clear consciousness, and discharged on day 7 post-operation.

ECG artifacts can be classified into two main groups: physiological and nonphysiological artifacts. Some causes of physi-

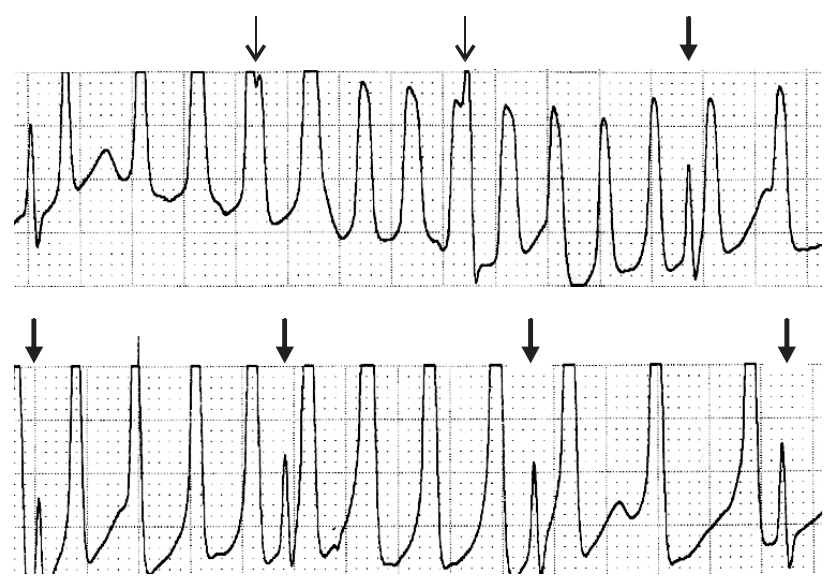

Fig. 1. Rhythm strips on both events. It relevantly showing notch sign (arrow) and spike sign (bold arrow).

Corresponding author: Ji Yeon Kim, M.D., Ph.D., Department of Anesthesiology and Pain Medicine, Inje University, Ilsan Paik Hospital, 2240, Daewha-dong, Ilsanseo-gu, Goyang 411-706, Korea. Tel: 82-31-910-7187, Fax: 82-31-910-7184, E-mail: jy67925@naver.com

(c) This is an open-access article distributed under the terms of the Creative Commons Attribution Non-Commercial License (http:// creativecommons.org/licenses/by-nc/3.0/), which permits unrestricted non-commercial use, distribution, and reproduction in any medium, provided the original work is properly cited. 
ological artifacts include muscular activity and the movement of patients [2]. Narrow, rapid, and spiky electromyographic signals may present on the ECG due to muscular activity, but these signals are mostly filtered by recent ECG monitors. Artifacts due to movement result from the ECG electrode being attached to the patient's skin; as it actively generates the potential difference between the interior and exterior of the skin. Stretching the skin changes the degree of skin potential, which can cause various artifacts on the ECG [3]. Epidermal stretch-induced voltage changes are the primary cause of motion artifacts, and such artifacts are not filtered [2]. In our case, a VT-like abnormal rhythm appeared on the ECG when the operator was conducting traction and dissection at the upper pole of the thyroid. Therefore, an epidermal stretch-induced voltage change due to the traction of the skin during traction of the huge thyroid can not be ruled out as causing the artifact.

Common causes for nonphysiological artifacts include insufficient electrode gel or contamination of the electrode by betadine [2]. However, there is a low possibility of the abnormal signal being caused by insufficient gel or contamination, because the abnormal ECG was presented in the middle of surgery and a protective film was applied to prevent electrode contamination.

While using monopolar electrocauterization, a faulty ground or electrical noise input may be another cause of nonphysiological artifacts. In this case, the electrocauterizer grounding pad was attached to the left peroneal surface, and there was no abnormal manifestation on the ECG at cauterization prior to the occurrence of the abnormal rhythm; hence, there is a low possibility of artifact due to these factors.

Also, thyrotoxic crisis could be a possibility. However, although there was an increase in the heartbeat on ECG, at that time BP was normal; this situation differs from thyrotoxic crisis, where systolic hypertension under a high-output state with a widening of pulse pressure is observed. Furthermore, as most of the thyroid gland was dissected, there is a low possibility that an abrupt input of thyroid hormone into the blood vessel could have caused VT.

Huang et al. [4] suggested a diagnostic algorithm for pseudoVT caused by a tremor-induced artifact. For a suspected manifestation of VT on ECG, pseudo-VT can be diagnosed when one of the following signs is observed: "sinus sign", where the sinus rhythm and normal P, QRS, and T wave are observed in a single limb lead (leads I, II, III); "spike sign", where a regular or irregular tiny spike is observed on the QRS complex; and "notch sign", where a notch is observed on the QRS-like complex with sinus rhythm cycle. In this case, the spike sign and notch sign were observed, and pseudo-VT caused by an artifact was diagnosed accordingly.

Arrhythmia during anesthesia is a frequently experienced manifestation. Especially, VT may cause a serious hemodynamic abnormality, immediate diagnosis and treatment are important. However, a normal rhythm can be construed as a serious arrhythmia due to the artifact. Therefore, it is crucial to consider the possibility of unnecessary and potentially fatal treatment at all times. Srikureja et al. [5] reported that artifacts as a causative factor always should be taken into account when a hemodynamically stable, asymptomatic patient presents with an abnormality on the ECG and confirmation of each lead on 12-lead ECG at the time of the episode, and checking the basic pulse.

In conclusion, when suspecting arrhythmia during anesthesia, it is essential to confirm the uniform occurrence of arrhythmia on other confirmable leads, and the diagnostic approach should include collecting clinical evidences such as the wave form on the pulse oximeter, PR and BP at the time of the event.

\section{References}

1. Knight BP, Pelosi F, Michaud GF, Stickberger SA, Morady F. Clinical consequences of electrocardiographic artifact mimicking ventricular tachycardia. N Engl J Med 1999; 341: 1270-4.

2. Chase C, Brady WJ. Artifactual electrocardiographic change mimicking clinical abnormality on the ECG. Am J Emerg Med 2000; $18: 321-6$.

3. De Talhouet H, Webster JG. The origin of skin-stretch caused motion artifacts under electrodes. Physiol Meas 1996; 17: 81-93.

4. Huang CY, Shan DE, Lai CH, Fong MC, Huang PS, Huang HH, et al. An accurate electrocardiographic algorithm for differentiation of tremor-induced pseudo-ventricular tachycardia and true ventricular tachycardia. Int J Cardiol 2006; 111: 163-5.

5. Srikureja W, Darbar D, Reeder GS. Tremor-induced ECG artifact mimicking ventricular tachycardia. Circulation 2000; 102: 1337-8. 